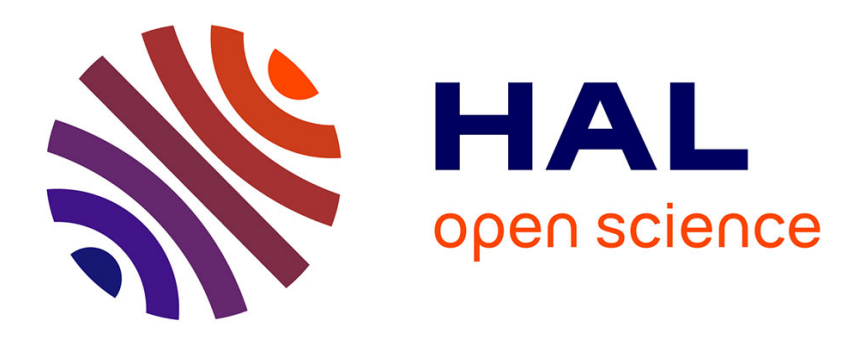

\title{
Combinatorics of the Gauss digitization under translation in $2 \mathrm{D}$
}

Etienne Baudrier, Loïc Mazo

\section{To cite this version:}

Etienne Baudrier, Loïc Mazo. Combinatorics of the Gauss digitization under translation in 2D. Journal of Mathematical Imaging and Vision, 2018, 61, pp.224-236. 10.1007/s10851-018-0846-5 . hal01853991

\section{HAL Id: hal-01853991 \\ https://hal.science/hal-01853991}

Submitted on 6 Aug 2018

HAL is a multi-disciplinary open access archive for the deposit and dissemination of scientific research documents, whether they are published or not. The documents may come from teaching and research institutions in France or abroad, or from public or private research centers.
L'archive ouverte pluridisciplinaire HAL, est destinée au dépôt et à la diffusion de documents scientifiques de niveau recherche, publiés ou non, émanant des établissements d'enseignement et de recherche français ou étrangers, des laboratoires publics ou privés. 


\title{
Combinatorics of the Gauss digitization under translation in $2 \mathrm{D}$
}

\author{
Étienne Baudrier, Loïc Mazo \\ 300 Bd Sébastien Brant - CS 10413 - 67412 ILLKIRCH, FRANCE
}

August 6, 2018

\begin{abstract}
The action of a translation on a continuous object before its digitization generates several digital objects. This paper focuses on the combinatorics of the generated digital objects up to integer translations. In the general case, a worst-case upper bound is exhibited and proved to be reached on an example. Another upper bound is also proposed by making a link between the number of the digital objects and the boundary curve, through its self-intersections on the torus. An upper bound, quadratic in digital perimeter, is then derived in the convex case and eventually an asymptotic upper bound, quadratic in the grid resolution, is exhibited in the polygonal case. A few significant examples finish the paper.
\end{abstract}

\section{Introduction}

For a given grid step and a given digitization method, a planar object produces several digital objects in function of its position on the grid. The digitally estimated object characteristics depend on the obtained digital object. The performances of the digital estimators on a set of digital objects coming from the same continuous object up to a translation is an information that can be useful for the digital-estimator assessment. Nevertheless, it can only rely on a knowledge of the combinatorics of the digitization up to a translation and on an efficient generator of translated digital objects. Thereby, as a first step, a study of the digitization combinatorics under translation is presented in this paper.

The link between geometrical primitives and their digitizations is widely studied. For instance, for the straight segments, it is possible to give the digitization in function of the segment slope and offset. Then, for a digital straight segment, its preimage by this latter function forms the parameter set of all the continuous straight segments whose digitization is equal to the given digital segment. The preimage is used for digital straight segment generation, combinatorics and recognition [3. The preimage has been generalized to polygons [4. Nevertheless, the study proposed here comes from another point of view: instead of focusing on the continuous objects giving the same digital object, 
we study the set of digital objects given by a single continuous object under translation.

A similar approach can be found in the studies of the generation and combinatorics of the disc digitization set in function of its radius and center position [5, 11, 20, 17, 19, 14, 8, 9, 10] and to the combinatorics of the strictly convex sets $[6]$.

In the general case, the digital-object set can be seen as the consequence (through a digitization) of the action of the rigid motion group on the continuous object ${ }^{1}$. In this article, we limit ourselves to the simplest digitization scheme: the Gauss digitization. The Gauss scheme just performs the intersection of the object with the set of the integer points, $\mathbb{Z}^{2}$. Then, a function, so-called dual, linking the translation group and the produced digital objects is used by the authors to study the digital-object set up to a translation, for function graphs in [1] and for planar object in [13. In the latter case, the dual has been proved to be piecewise constant in function of the translation parameters. This paper focuses on the dual combinatorics. The results on the general and the convex case have already been presented in [13. The paper extends these results to the polygonal case.

The paper layout is the following: two upper bounds are given for the number of digitizations of a planar solid object whose boundary is a Jordan curve. The first one is expressed in terms of the number of grid cells crossed by the boundary and the second one in terms of the number of intersections when plotting the continuous-object boundary on the torus $\mathbb{R}^{2} / \mathbb{Z}^{2}$. The latter bound is proved to be quadratic in the convex case and to be asymptotically quadratic in the resolution in the polygon case. Some examples are provided in order to compare the two upper bounds both in the convex and the non-convex cases. A conclusion and some perspectives end the paper.

\section{Background}

Let us consider a connected compact set $S$ in $\mathbb{R}^{2}$ whose boundary is a simple closed (Jordan) curve $\Gamma$. Thanks to the Jordan curve theorem, we may assume a continuous map $f: \mathbb{R}^{2} \rightarrow \mathbb{R}$ such that $\Gamma$ and $S$ are implicitly defined by

$$
\begin{gathered}
\Gamma=\left\{f(z)=0 \mid z \in \mathbb{R}^{2}\right\} \\
S=\left\{z \in \mathbb{R}^{2} \mid f(z) \leq 0\right\} .
\end{gathered}
$$

The common methods to model the digitization of the set $S$ are closely related to each other. In this paper, we assume a Gauss digitization. This method associates to the set $S$ the grid points that lie inside $S$, that is $S \cap \mathbb{Z}^{2}$, or, equivalently, the binary image defined on $\mathbb{Z}^{2}$ whose 1 's are the points inside $S$.

We are interested in the variability of the Gauss digitization when the group of the translations acts on $S$, that is on the sets $(u+S) \cap \mathbb{Z}^{2}, u \in \mathbb{R}^{2}$. In

\footnotetext{
${ }^{1}$ Remark that group actions on a digital object (i.e. a digital object moved in another digital object) were studied for the group of rigid motions in e.g. [16, 15, 17. In these works, the digitization step is out of the scope and beside, the case of the translations is obvious.
} 
this paper, we focus on the combinatorial aspects of this variance. Of course, the variance has to be understood "up to integer translations". This is the reason why we defined in a previous paper [13] the dual by translation ${ }^{2}$ of the digitizations of $S$ as a set-valued function $\varphi_{S}$ defined on the torus $\mathbb{T}=\mathbb{R}^{2} / \mathbb{Z}^{2}$ which maps each point $t \in \mathbb{T}$ to the produced digital object, up to integer translations, of $u+S$ where the vector $u$ is any representative of $t$ in $\mathbb{R}^{2}$. Let us pick a representative in each class of $\mathbb{T}$ so as to form a connected set $M$ called the structuring element. We note $C$ the symmetric of $M$ with respect to the origin: $C=-M$. The family of sets $p+C, p \in \mathbb{Z}^{2}$, is a tiling of $\mathbb{R}^{2}$. For instance, we can take $M=[0,1)^{2}, C=(-1,0]^{2}$. From now on, to simplify the notations, we identify $t \in \mathbb{T}$ with its representative in $M$ and the subsets of $\mathbb{Z}^{2}$ with their orbits for the action of the integer translations so we can write $\varphi_{S}(t)=(t+S) \cap \mathbb{Z}^{2}$. For any point or set $X$ in $\mathbb{R}^{2}$, we denote by $\operatorname{proj}(X)$ its projection on the quotient space $\mathbb{T}$. We proved in [12] that the plot of the curve $\Gamma$ on the torus $\mathbb{T}, \operatorname{proj}(\Gamma)$, delineates regions on which the dual function $\varphi_{S}$ is constant.

We define the grid boundary $\mathcal{B}$ as the set of grid points that lie in the (morphological) dilation of the boundary $\Gamma$ of $S$ by the structuring element $M$ :

$$
\mathcal{B}=(\Gamma \oplus M) \cap \mathbb{Z}^{2},
$$

where $\oplus$ denotes the Minkowski sum. The set $\mathcal{B}$ contains all the points of $\mathbb{Z}^{2}$ whose membership to the digitization may change when the set $S$ is shifted by a vector $u \in M$. Nevertheless, since $\mathbb{R}^{2}$ is connected, $M$ is not open so there may exist some points in $\mathcal{B} \cap S$ not liable to change, namely those points $p$ in $\mathcal{B}$ for which $p+C \subseteq S$ (see Fig. 1). That is why we have in fact to consider the toggling boundary $\mathfrak{B}$ as the set of grid points whose membership effectively toggle for some translation by a vector $u \in M$ :

$$
\mathfrak{B}=\mathcal{B} \backslash\left\{p \in \mathbb{Z}^{2} \mid p+C \subseteq S\right\} .
$$

The set $S \cap \mathbb{Z}^{2} \backslash \mathfrak{B}$ of the grid points that are in any digitization of $u+S, u \in M$, is called the digitization core.

Finally, for any $p \in \mathbb{Z}^{2}$, we set

$$
\Gamma_{p}=-p+(\Gamma \cap(p+C))=(-p+\Gamma) \cap C,
$$

so $\Gamma_{p}=\emptyset$ if $p \notin \mathcal{B}$, and we denote by $\mathbf{1}_{p}$ the indicator function of the set $(-p+S) \cap C$, so $\mathbf{1}_{p}$ is not constant iff $p \in \mathfrak{B}$. Then,

$$
\varphi_{S}(t)=\left\{p \in \mathbb{Z}^{2} \mid \mathbf{1}_{p}(-t)=1\right\} .
$$

In the rest of the article, we use the notation $|E|$ for the cardinal of a set $E$ $(|E| \in \mathbb{N} \cup\{\infty\}), \operatorname{CC}(A)$ for the family of the connected components of a subset $A$ of $\mathbb{R}^{2}$ and $I \sqcup J$ for the disjoint union of the sets $I$ and $J$.

\footnotetext{
2 The "dual" term is an analogy with the algebraic dual because our construction transforms a set of binary images on a discrete set $\left(\mathbb{Z}^{2}\right)$ in a labeled image on a set of transformations (the translations).
} 


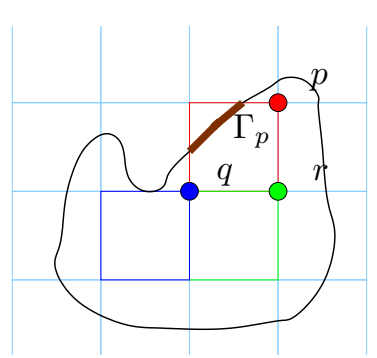

(a)

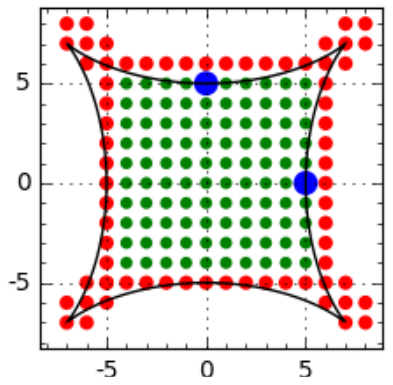

(b)

Figure 1: (Color online) (a) A Jordan curve $\Gamma$ and a set of tiles $z+C, z \in \mathbb{Z}^{2}$ and $C=(-1,0]^{2}$. The point $p$ is in $\mathfrak{B}$ for the square $p+C$ intersects both $\Gamma$ and $\mathbb{Z}^{2} \backslash S$; the point $q$ is in $\mathcal{B} \backslash \mathfrak{B}$ for the square $q+C$ intersects the boundary $\Gamma$ but is included in $S$; the point $r$ is in the digitization core for the square $r+C$ is included in $S$ and does not intersect $\Gamma$ (b) Black: a Jordan curve, medium red discs: the toggling boundary, big blue discs: two points that lie in the grid boundary but that cannot change their membership, small green discs: the digitization core.

In the following section, upper bounds are given for the number of digitizations up to a translation. The first upper bound is naive. For the second one, the idea is to link the digitization number to the number of the intersection of the translated curves $\Gamma_{p}$. Indeed, the dual induces a partition of the torus whose cells frontiers are arcs of the curve $\operatorname{proj}(\Gamma)$. Then, we bound from above the partition size by counting the number of curve intersections in $\operatorname{proj}(\Gamma)$.

\section{The general case}

\subsection{Bounding up by counting the crossed tiles}

Since the grid boundary $\mathcal{B}$ contains any integer point whose value may change when shifting the set $S$, we have a first, obvious, upper bound on the number of Gauss digitizations given by $2^{|\mathcal{B}|}$. As $|\mathcal{B}|$ is also the number of tiles $p+C$, $p \in \mathbb{Z}^{2}$, crossed by the frontier of $S$, we can state the following proposition.

Proposition 3.1. The number of Gauss digitizations, up to integer translations, is upper bounded by $2^{\mathrm{a}(\Gamma)}$ where $\mathrm{a}(\Gamma)$ is the number of tiles crossed by $\Gamma$.

Generally, the digitization enumeration provided by Prop. 3.1 includes false positives and multiple counts. For instance, the grid boundary of a circle with diameter 1.7 involves 4 to 8 pixels, depending on the grid position, which gives an upper bound according to Prop. 3.1 equals to 16, while there exists only 8 digitizations (see Fig 2). Nevertheless, from any set, it is possible to build a new set that avoids false positives by replacing the initial boundary by a family 


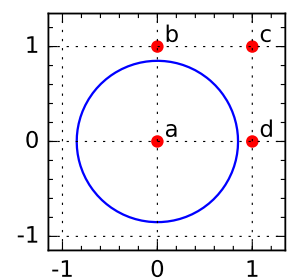

(a)



(b)

Figure 2: (a) A circle with diameter 1.7 and the four pixels in the toggling boundary (which is equal to the grid boundary): a, b, c, d. (b) The (flatten) dual of the closed disc bounded by the circle. Among the $16=2^{4}$ potential digitizations provided by the 4-pixels a, b, c, d, three of them does not actually appear $(\emptyset$, ac and bd) and some others appear multiple times (the four singular digitizations, which are obviously congruent, and the vertical and horizontal pairs which each appear twice. Eventually, there are only 8 digitizations (and actually the (sewed) dual is divided into 8 regions).

of Hilbert curves and it is possible to extend the set to prevent multiple counts so that the theoretical upper bound $2^{|\mathfrak{B}|}$ is obtained (see Appendix B).

\subsection{Bounding up by counting the intersections}

In Sec. 3.2, we assume a parametrization of $\Gamma$. It induces an order on the points of the curve $\Gamma$ (for $\Gamma$ is simple) that is used in the proof of the following proposition.

The dual $\varphi_{S}$ can be regarded as the projection, on the torus $\mathbb{T}$ of a finite labeled partition of the tile $C$ (whose cells need not be connected). For the order by refinement on the partitions, this partition is lower bounded by the infimum $\bigwedge_{p \in \mathfrak{B}} \mathcal{P}_{p}$ of the binary partitions $\mathcal{P}_{p}$ associated to the indicator functions $\mathbf{1}_{p}, p \in$ $\mathfrak{B}$. Since some cells of $\bigwedge_{p \in \mathfrak{B}} \mathcal{P}_{p}$ need to be merged whenever the corresponding digitizations are equivalent up to an integer translation, the size of the partition $\bigwedge_{p \in \mathfrak{B}} \mathcal{P}_{p}$ is generally greater than the number of digitizations. For instance, the circle of Fig 2 induces 4 binary partitions of the unit square by the 4 quarters of circle. The infimum of these four partitions yields the partition in 13 classes shown in Fig $2 \mathrm{~b}$ which is finer than the partition in the 8 classes of digitization (up to integer translations).

The next proposition proposes an upper bound on the size of the partition $\bigwedge_{p \in \mathfrak{B}} \mathcal{P}_{p}$ - and thereby an upper bound to the number of digitizations - by inductively counting the intersections between the curves $\Gamma_{p}, p \in \mathfrak{B}$. The idea is to count the partition cells created when adding a curve $\Gamma_{p}$. To do this we count the intersection of $\Gamma_{p}$ with the already added curves. Nevertheless, such an intersection can be with one (Fig. 3b) or more curves (Fig. 3c) and can be a singleton (Fig. 3c), a set of several points (Fig. 3b) or an arc (Fig. 3d). Then, let us specify how we handle intersections in this context. 
Definition 1. Let $\mathfrak{B}=\left\{b_{1}, \cdots, b_{i}, \cdots, b_{n}\right\}, n \geq 1$. Let $k \in[2, n]$. Then, we set:

$$
\begin{aligned}
& \text { - } \text { inter }_{\gamma, I}=\operatorname{CC}\left(\gamma \cap\left(\bigcap_{i \in I} \Gamma_{b_{i}} \backslash \bigcup_{j \in J} \Gamma_{b_{j}}\right)\right), \\
& \text { where } \gamma \in \operatorname{CC}\left(\Gamma_{b_{k}}\right) \text { and } I \sqcup J=[1, k-1] ; \\
& \text { - } \# \text { inter }_{k}=\sum_{\substack{\gamma \in \operatorname{CC}\left(\Gamma_{b_{k}}\right) \\
\emptyset \subset I \subseteq[1, k-1]}} w_{I}\left|\operatorname{inter}_{\gamma, I}\right|, \\
& \text { where } w_{I}=\min (|I|, 2) .
\end{aligned}
$$

Note that the components of all the inter $r_{\gamma, I}$ are two by two disjoint and that \# inter $_{k}$ may be infinite. The set inter ${ }_{\gamma, I}$ stands for the set of the connected intersections of the arc $\gamma \subseteq \Gamma_{b_{k}}$ with the curves whose indexes are in a subset $I$, excluding any other curve $\Gamma_{b_{i}}$ whose index is not in $I$. The necessity to have all the index subsets comes from the fact that intersections between multiple curves can occur. In Fig. $3 \mathrm{~b}$ where $\gamma$ is the green curve $\Gamma_{b_{2}}, I=\{1\}$ and $J=\emptyset$, inter $_{\gamma, I}$ contains four connected components, the four intersection points. In Fig. 3c where $\gamma$ is the brown curve $\Gamma_{b_{3}}$, we consider that the intersection of $\gamma$ with the blue curve alone, or with the green curve alone, is empty but the intersection of $\gamma$ with both curves has one connected component, the triple intersection point. Nevertheless, in \# inter 3 , the weighted sum of the cardinals, inter $_{\Gamma_{b_{3}},\{1,2\}}$ is counted twice (the weights are limited to 2 for we show in the proof that if the number of intersections is finite, an intersection creates at most 2 new cells). In Fig. $3 \mathrm{~d}$ where $\gamma$ is the red curve $\Gamma_{b_{4}}$, the curve $\gamma$ has a single intersection with the blue curve, which is an arc, and a single intersection that involves the three other curves, which is then counted twice.

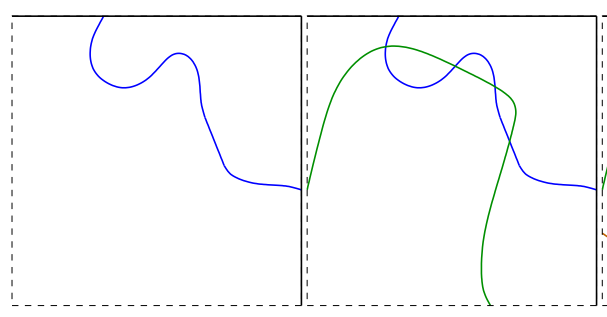

(a) (b)



(c)



(d)

Figure 3: (Color online) (a) Blue: $\Gamma_{b_{1}}$. (b) Green: $\Gamma_{b_{2}}$; \# inter $\operatorname{inter}_{\Gamma_{b_{2}},\{1\}}=|\operatorname{inter}|=4$. (c) Brown: $\Gamma_{b_{3}}$; \# inter inter $_{\Gamma_{b_{3}},\{1\}}|+| \operatorname{inter}_{\Gamma_{b_{3}},\{2\}}|+2| \operatorname{inter}_{\Gamma_{b_{3}},\{1,2\}} \mid=0+0+2=$ 2. (d) Red: $\Gamma_{b_{4}}$; \# inter $_{4}=\left|\operatorname{inter}_{\Gamma_{b_{4}},\{1\}}\right|+2\left|\operatorname{inter}_{\Gamma_{b_{4}},\{1,2,3\}}\right|=2+2=4$.

We are now able to state the proposition that relates the number of digitizations and the number of intersections \# inter $_{k}$. 
Proposition 3.2. The size of the partition $\bigwedge_{p \in \mathcal{B}} \mathcal{P}_{p}$ is upper bounded by

$$
2+\sum_{k=2}^{n} \# \operatorname{inter}_{k}+\left|\mathrm{CC}\left(\Gamma_{b_{k}}\right)\right|
$$

Proof. The proposition is proved by a finite induction: we show that the size of the partition $\bigwedge_{i=1}^{m} \mathcal{P}_{b_{i}}$ is upper bounded by $2+\sum_{k=2}^{m} \# \operatorname{inter}_{k}+\left|\mathrm{CC}\left(\Gamma_{b_{k}}\right)\right|$ for $m=1,2, \cdots, n$. For $m=1$, the result is obvious since, for any $p \in \mathfrak{B}, \mathcal{P}_{p}$ is a binary partition. Let $m>1$. We assume that the number of cells of the partition $\bigwedge_{i=1}^{m-1} \mathcal{P}_{b_{i}}$ is upper bounded by $2+\sum_{k=2}^{m-1} \#$ inter $_{k}+\left|\mathrm{CC}\left(\Gamma_{b_{k}}\right)\right|$. The cells of $\bigwedge_{i=1}^{m-1} \mathcal{P}_{b_{i}}$ which are included in one of the two cells of $\mathcal{P}_{b_{m}}$, namely the sets $\left(\mathbf{1}_{b_{m}}=1\right)$ and $\left(\mathbf{1}_{b_{m}}=0\right)$, stay unchanged in the partition $\bigwedge_{i=1}^{m} \mathcal{P}_{b_{i}}$. Conversely, the cells of $\bigwedge_{i=1}^{m-1} \mathcal{P}_{b_{i}}$ that are intersected by both $\left(\mathbf{1}_{b_{m}}=0\right)$ and $\left(\mathbf{1}_{b_{m}}=1\right)$, which obviously are cells intersected by $\Gamma_{b_{m}}$, are each divided in two new cells. Thereby, the number $N_{m}$ of new cells is upper bounded by $N_{m}^{\prime}$, the number of cells in $\bigwedge_{i=1}^{m-1} \mathcal{P}_{b_{i}}$ intersected by $\Gamma_{b_{m}}$. Besides, the partition $\bigwedge_{i=1}^{m-1} \mathcal{P}_{b_{i}}$ of the tile $C$ induces a partition $\mathcal{Q}$ of $\Gamma_{b_{m}}$, as a subset of $C$, with $N_{m}^{\prime}$ cells (these cells of $\Gamma_{b_{m}}$ need not be connected). Then, the idea of the proof is to map each cell of $\mathcal{Q}$ to its supremum, for the order induced by the parametrization of $\Gamma$, namely to the intersection, as defined in Def. 1 its supremum belongs to, or to the empty set when no such intersection exists. Then, though this mapping is not one-to-one, a careful examination of the different cases will permit to conclude that $N_{m}^{\prime}$ is upper bounded by \# inter ${ }_{m}+\left|\mathrm{CC}\left(\Gamma_{b_{m}}\right)\right|$. The configurations studied in the proof are illustrated by Fig. 4 .

Let $\gamma$ be a connected component of $\Gamma_{b_{m}}$ and $s_{\infty}$ be the supremum of $\gamma$ for the order induced by the parametrization of $\Gamma$. Let $\mathcal{Q}_{\gamma}$ be the restriction of $\mathcal{Q}$ to $\gamma$. We set inter $\gamma=\bigcup_{\emptyset \subset I \subseteq[1, m-1]}$ inter $_{\gamma, I}$, and we assume the following facts that will be proved further:

a) each component in inter $\gamma$ is included in a cell of $\mathcal{Q}_{\gamma}$;

b) if a cell of $\mathcal{Q}_{\gamma}$ has a supremum $s$ distinct from $s_{\infty}$, then $s$ belongs to some component of inter $r_{\gamma}$

c) if a cell $K_{0}$ of $\mathcal{Q}_{\gamma}$ has its supremum $s$ in some component $K$ of inter ${ }_{\gamma}$ and $s \notin K_{0}$, then $s$ is the infimum of $K$.

d) if two cells $K_{1}, K_{2}$ of $\mathcal{Q}_{\gamma}$ have the same supremum $s$ and $s \notin K_{1} \cup K 2$, then inter $\gamma$ has infinitely many components;

e) if two cells $K_{1}, K_{2}$ of $\mathcal{Q}_{\gamma}$ have their supremums $s_{1}, s_{2}$ in the same component of inter $\gamma$ and $s_{1} \in K_{1}$ while $s_{2} \notin K 2$, then either $s_{2}=s_{\infty}$ and $s_{\infty}$ belongs to some component of inter $\gamma$, or $s_{2}$ belongs to at least two curves $\Gamma_{b_{i}}$ and $\Gamma_{b_{j}}$ where $i, j<m$.

In the configuration of Fig $4 \mathrm{ff}$, Fact $\mathrm{c}$ happens for the cyan and the yellow cells if the magenta cell is topologically closed in the tile $C$ and if the number of 
oscillations of the curve near the center point is infinite as in Fig $4 \mathrm{~h}$. In the same configuration, Fact d happens for the cyan and the yellow cells if the cyan cell is topologically closed while the magenta cell is open (thus, forgetting Fig 4a). Indeed, in such case, the component of inter ${ }_{\gamma}$ right of the center point is closed and included in the cyan cell while the supremum of the yellow cell is the center point which belongs to the cyan cell.

Then, with these five assumptions, we deduce the desired upper bound as follows. We map each cell of $\mathcal{Q}_{\gamma}$ to the component of inter ${ }_{\gamma}$ its supremum belongs to, if any. The other cells are mapped on the empty set. We denote by $\psi$ this mapping.

- If a component $K$ of inter ${ }_{\gamma}$ has three, or more, preimages by the mapping $\psi$, then from Fact a, and because $\mathcal{Q}_{\gamma}$ is a partition, we derive that at least two of these preimages do not contain their supremums. Then, from Fact c, we get that these supremums are equal (to the infimum of $K$ ). Finally, from Fact d, we conclude that $\left|\operatorname{inter}_{\gamma}\right|=\infty$ which implies that \# inter ${ }_{m}$ is also infinite. So, $N_{m}^{\prime}$ is obviously upper bounded by \# $\operatorname{inter}_{m}+\left|\mathrm{CC}\left(\Gamma_{b_{m}}\right)\right|$.

Alike, if the empty set has two, or more, preimages by $\psi$, we derive from Fact $\mathrm{b}$ and the definition of $\psi$ that the supremum of these preimages is $s_{\infty}$ and that $s_{\infty}$ does not belong to any component of inter ${ }_{\gamma}$. If $s_{\infty} \in C$, since $\gamma \backslash \bigcup_{K \in \text { inter }_{\gamma}} K=\gamma \backslash \bigcup_{i<m} \Gamma_{b_{i}}$ is an open set of $C$, all the indicator functions $\mathbf{1}_{b_{i}}$ would be constant in a neighborhood of $s_{\infty}$ which contradicts the assumption that $s_{\infty}$ is the supremum of two cells of $\mathcal{Q}_{\gamma}$. So, $s_{\infty} \notin C$ and we conclude as above by invoking Fact $d$.

- If a component $K$ of inter $_{\gamma}$ has exactly two preimages $K_{1}$ and $K_{2}$ by the mapping $\psi$ and if Fact $\mathrm{d}$ does not apply (otherwise, we conclude straightforwardly as above), then, from Fact e, and the very definition of

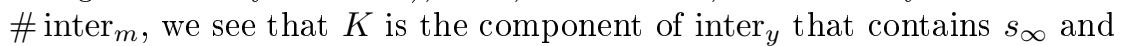
the empty set has no preimage, or the weight of $K$ in $\# \operatorname{inter}_{m}$ is 2 , which corresponds to the number of its preimages.

We readily conclude that the number of cells of $\mathcal{Q}_{\gamma}$ is upper bounded by $1+\sum_{\emptyset \subset I \subseteq[1, m-1]} w_{I} \mid$ inter $_{\gamma, I} \mid$ where $w_{I}=\min (2,|I|)$. By summing on all the connected components of $\Gamma_{b_{m}}$, we derive that $N_{m}^{\prime}$, the number of cells of $\mathcal{Q}$, is upper bounded by \# inter $_{m}+\left|\mathrm{CC}\left(\Gamma_{b_{m}}\right)\right|$ which achieves the proof.

Let us now prove the five facts stated above.

a) We demonstrate Fact a by contradiction. Suppose it exists $K$ in inter ${ }_{\gamma, I}$, $I \subseteq[1, m-1]$, and two distinct cells $K_{1}, K_{2}$ of $\bigwedge_{i=1}^{m-1} \mathcal{P}_{b_{i}}$ such that $K \cap K_{1} \neq \emptyset$ and $K \cap K_{2} \neq \emptyset$. Let $c_{1} \in K \cap K_{1}$ and $c_{2} \in K \cap K_{2}$. Since $K_{1} \neq K_{2}$, there exists $i \in[1, m-1]$ such that $\mathbf{1}_{b_{i}}\left(c_{1}\right) \neq \mathbf{1}_{b_{i}}\left(c_{2}\right)$. Then, the segment $\left[c_{1}, c_{2}\right]$ in $\gamma$ contains a point $c_{3} \in \Gamma_{b_{i}}$ which also belongs to $K$ for $K$ is connected. Then, on the one hand $i \in I$ for $c_{3} \in \Gamma_{b_{i}} \cap K$ and, on the other hand, $c_{1}$, or $c_{2}$, is in $\left(\mathbf{1}_{b_{i}}=0\right)$, that is $i \notin I$. Contradiction.

b) Suppose that the supremum $s$ of the cell $K$ of $\mathcal{Q}_{\gamma}$ is not in any component of inter $_{m}$. Then, for any $i \in[1, m-1], s \notin \Gamma_{b_{i}}$. In other words, for any 


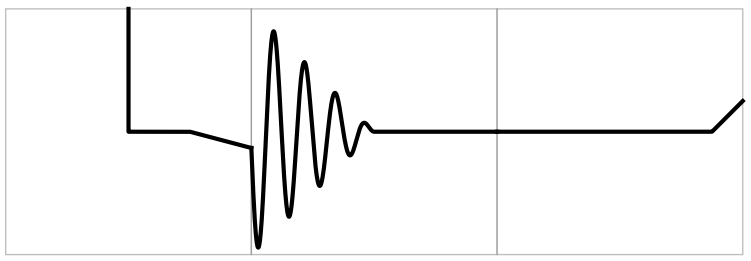

(a)

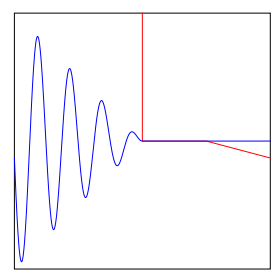

(b)

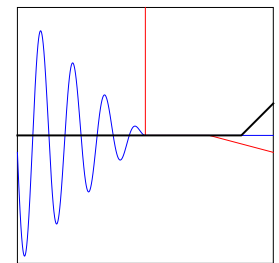

(d)

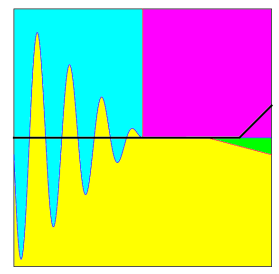

(f)

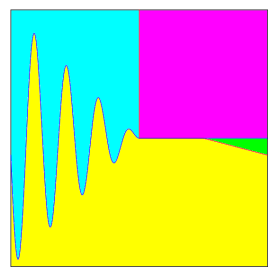

(c)

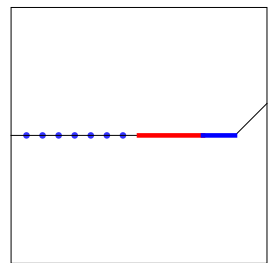

(e)

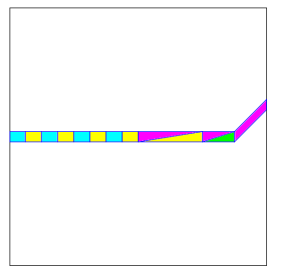

$(\mathrm{g})$

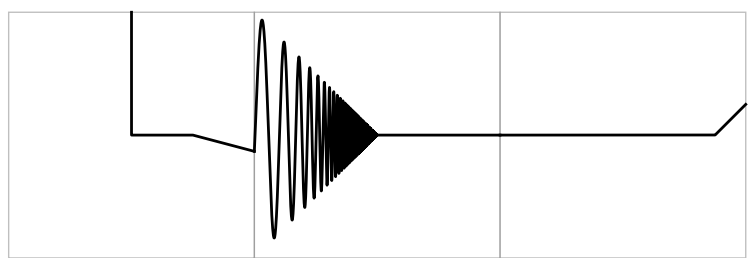

(h)

Figure 4: (a) From left to right, the three first grid squares crossed by a Jordan curve $\Gamma$. (b) The two first curve segments plotted on the tile $C: \Gamma_{b_{1}}, \Gamma_{b_{2}}$. (c) The partition $\mathcal{P}_{b_{1}} \wedge \mathcal{P}_{b_{2}}$. (d) The third segment $\gamma=\Gamma_{b_{3}}$ of the curve $\Gamma$ is plotted on $C$. (e) The 9 intersections (connected components) in inter $\gamma$. (f)-(g) The partition $\mathcal{Q}$ induced by $\mathcal{P}_{b_{1}} \wedge \mathcal{P}_{b_{2}}$ on $\gamma$. Note that for two cells of $\mathcal{Q}$ (in yellow and green), the number of connected components depends on the location of the shape $S$ : if $\Gamma$ rotates clockwise the yellow cell has 5 components and the green cell one component; otherwise, the yellow cell has 4 components and the green cell does not exist (and the magenta cell is much bigger). (h) By modifying the curve $\Gamma_{b_{2}}$, replacing the damped sinusoid of $x$ by a damped sinusoid of $1 / x$, we obtain an example where the number of intersections in inter $_{\gamma}$ is infinite. 
$i \in[1, m-1], s$ is in the open set $\left(\mathbf{1}_{b_{i}}=0\right)$ or in the interior of $\left(\mathbf{1}_{b_{i}}=1\right)$. Thus, there exists an open neighborhood $U$ of $s$ in the tile $C$ which does not intersect any curve $\Gamma_{b_{i}}, 1 \leq i<m$. Thereby, $U$ is included in a cell of the partition $\bigwedge_{i=1}^{m-1} \mathcal{P}_{b_{i}}$. Since $s=\sup (K)$, there is no point $t$ in $\gamma \cap U$ such that $t>s$. Thus, $s=s_{\infty}$.

c) Let $K_{0}$ be a cell of $\mathcal{Q}_{\gamma}$ whose supremum $s$ is in a component $K \in$ inter $_{\gamma}$ and not in $K_{0}$. Let $K_{1}$ be the cell of $\mathcal{Q}_{\gamma}$ which includes the component $K$ (Fact a). Since $s \notin K_{0}$, then $K_{1} \neq K_{0}$ and $s$ is a limit point of $K_{0}$. If $s$ is not the infimum of $K$ then there exists an interval [u,s], with $u<s$, in $K \subseteq K_{1}$ (for $K$ is connected) and $s$ is not a limit point of $K_{0}$. Contradiction.

d) Let $K_{1}, K_{2}$ be two cells of $\mathcal{Q}_{\gamma}$ that share the same supremum $s$ that is not in $K_{1}$ nor in $K_{2}$. Then, $s$ a limit point for the two cells. Let $k \in[1, m-1]$ such that $\mathbf{1}_{b_{k}}$ takes two distinct values on $K_{1}$ and $K_{2}$. For instance, $K_{1} \subseteq\left(\mathbf{1}_{b_{k}}=0\right)$ and $K_{2} \subseteq\left(\mathbf{1}_{b_{k}}=1\right)$. Then, we can inductively build an infinite sequence $c_{1}<c_{2}<\cdots<c_{i}<\cdots<s$ such that $c_{2 i-1} \in K_{1}$ and $c_{2 i} \in K_{2}$ for any $i \geq 1$. In particular, we have $\mathbf{1}_{b_{k}}\left(c_{2 i-1}\right)=0$ and $\mathbf{1}_{b_{k}}\left(c_{2 i}\right)=1$ for any $i \geq 1$. Then, $c_{2 i-1} \notin \Gamma_{b_{k}}$ and $\left[c_{2 i-1}, c_{2 * i}\right]$ intersects $\Gamma_{b_{k}}$. Therefore, inter ${ }_{\gamma}$ contains infinitely may components between $c_{1}$ and $s$.

e) Let $K_{1}, K_{2}$ be two cells of $\mathcal{Q}_{\gamma}$ whose supremums $s_{1}, s_{2}$ belong to the same component $K$ of inter $\gamma$. Furthermore, assume $s_{1} \in K_{1}$ and $s_{2} \notin K_{2}$. Since $s_{2} \notin K_{2}, s_{2}$ is a limit point of $K_{2}$ and $s_{2}$ is the infimum of $K$ (Fact c). In particular, $s_{2} \leq s_{1}$. Let $I$ be the subset of $[1, m-1]$ such that $K$ is a component of inter ${ }_{\gamma, I}$. As in the proof of Fact b, we derive that there exists an open neighborhood $U$ of $s_{1}$ in $C$ which does not intersect any curve $\Gamma_{b_{i}}, i \notin I$. Suppose that $I$ is a singleton, say $I=\left\{b_{1}\right\}$. Then, taking a point in $K_{1} \cap U$ (recall that $s_{2}$ is a limit point of $K_{2}$ ), we found that necessarily $\mathbf{1}_{b_{1}}=0$ on $K_{2}$, while $\mathbf{1}_{b_{1}}=1$ on $K_{1}$ (for $s_{1} \in K \cap K_{1}$ ), and $\mathbf{1}_{b_{i}}, i \neq 1$, coincides on $K_{1}$ and $K_{2}$. If $s_{1} \neq s_{\infty}$, there exists a point $u$ in $\gamma \cap U$ greater than $s_{1}$. This point is not in $K_{1}$ for $s_{1}=\sup \left(K_{1}\right)$. Since $U$ does not intersect any curve $\Gamma_{b_{i}}, i \notin I$, we derive that $u \in K_{2}$ which contradicts $s_{2} \leq s_{1}$. Thus, either $s_{\infty}=s_{1} \in K$, or $I$ is not a singleton.

In the simplest case where the curve segments $\Gamma_{b_{m}}$ all have one connected component and where the intersections are sets of points belonging to at most two curves, Formula 1 reduces to $1+|\mathfrak{B}|+\mathfrak{I}$ where $\mathfrak{I}$ is the number of intersection points between the curve segments $\Gamma_{b_{m}}$. Furthermore, when projecting the partition $\bigwedge_{i=1}^{n} \mathcal{P}_{b_{i}}$ on the torus $\mathbb{T}$, the cells that touch the boundary of $C$ are identified two by two, which decreases the number of cells in the partition. Unfortunately, it is difficult to count these cells in the general case. 


\section{The convex case}

It seems plain that the structure of the dual should be simpler when the set $S$ is convex compared to a set with a winding boundary. We could even hope that the digitizations coincide with the cells of the partition $\bigwedge_{p \in \mathfrak{B}} \Gamma_{p}$, after sewing the boundary of the tile $C$, and with the connected regions of the torus $\mathbb{T}$ delineated by the curve $\Gamma$. Figure 5 annihilates this hope by exhibiting a convex object and one of its digitizations whose inverse image by the dual is not connected. Nevertheless, in this section we show that the structure of the sets

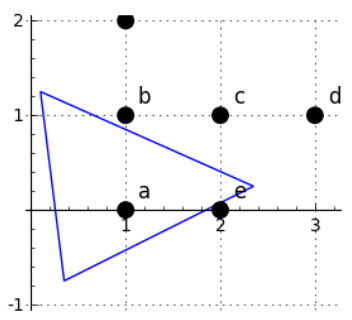

(a)

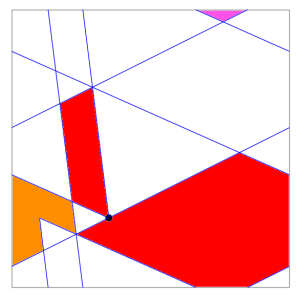

(b)

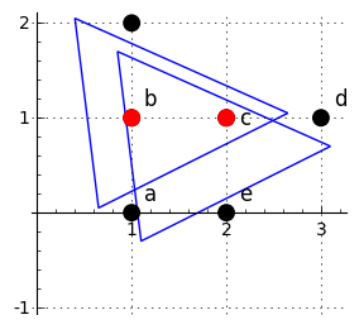

(c)

Figure 5: (Color on-line) (a) A triangle and its toggling boundary. (b) The dual of the (filled) triangle. The four colored regions correspond to the same digitization: the horizontal pair. Red region: cell bc of the partition $\bigwedge_{p \in \mathfrak{B}} \Gamma_{p}$. The red region is disconnected by the unique point of the cell abcd. Orange region: cell cd. Pink region: cell ae. (c) The configurations of the shifted triangle in the two red components. Since they are disconnected, it is not possible to continuously move to one configuration to the other without hurting a black toggling point. Note that the triangle could be slightly inflated so as to provide a strictly convex counterexample.

inter $_{\gamma, I}$ is simple when the set $S$ is strictly convex (its boundary does not include any segment) and permits to obtain a quadratic bound for the complexity of the dual in terms of the grid boundary size (to be compare to the exponential bound of Prop. 3.1.

Proposition 4.1. We assume a convex quadrilateral structuring element $M$. Let $n=|\mathfrak{B}|$. The number of Gauss digitizations of a strictly convex planar object up to a translation is upper bounded by

$$
4 n^{2}+4 n-6 \text {. }
$$

Proof. The proof invokes Lemma A.3 which is stated and proved in the appendix. Let $N$ be the digitization number. Thanks to Prop. 3.2 , we have

$$
N \leq 2+\sum_{m=2}^{n} \# \operatorname{inter}_{m}+\left|\mathrm{CC}\left(\Gamma_{b_{m}}\right)\right|
$$

From Lemma A.3 we derive that, for any $m \in[1, n], \gamma \in \mathrm{CC}\left(\Gamma_{b_{m}}\right), i \in[1, m-1]$,

$$
\sum_{I \ni i} \mid \text { inter }_{\gamma, I}|\leq 2| \mathrm{CC}\left(\Gamma_{b_{i}}\right) \mid
$$


Then,

$$
\# \operatorname{inter}_{m} \leq 2 \sum_{k=1}^{m-1}\left|\mathrm{CC}\left(\Gamma_{b_{k}}\right)\right| .
$$

Eventually, Eq (2) turns into

$$
\begin{aligned}
N & \leq 2+2 \sum_{m=2}^{n} \sum_{k=1}^{m-1}\left|\mathrm{CC}\left(\Gamma_{b_{k}}\right)\right|+\sum_{m=2}^{n}\left|\mathrm{CC}\left(\Gamma_{b_{m}}\right)\right| \\
& \leq 2\left(1+\sum_{k=1}^{n}(n-k+1)\left|\mathrm{CC}\left(\Gamma_{b_{k}}\right)\right|-\left|\mathrm{CC}\left(\Gamma_{b_{1}}\right)\right|\right) .
\end{aligned}
$$

It can easily be seen (from Lemma A.2 for instance) that, for any convex curve $\Gamma$ and any convex polygon $P$ with $c$ edges, the number of connected arcs in the intersection $\Gamma \cap P$ is upper bounded by $c$. Then, if we assume that the tile $C$ is a convex quadrilateral, we straightforwardly obtain the desired bound.

The term $4 n^{2}$ in Prop. 4.1 comes down to $n^{2}$ for sufficiently high resolutions because each curve $\Gamma_{b_{i}}$ then have just one connected component instead of possibly 4 in the general case. Then, when $S$ a disc of radius $r$, the result of Prop. 4.1 is close to the one in [8] which states that the number of digitizations of the disc is asymptotically $4 \pi r^{2}+O(r)$. As the ratio between the size of the grid boundary and the radius $r$ is 8 for the disc, our upper bound in function of the radius $r$ of the disc is asymptotically equivalent to $64 r^{2}$.

\section{The polygonal case}

The aim of this section is to give the asymptotic order of the digitization number of a polygon when the digitization step $h$ tends toward 0 . Since the upper bound given in Prop. 3.2 relies on the intersection combinatorics, the following propositions focus on the intersection number asymptotic estimation.

For a given affine function $y=\alpha x+\beta$, its values at integer points follow an arithmetical sequence. There is a classical result about its fractional part [18: if the common difference of the sequence is irrational, the fractional-part sequence is equidistributed over $[0,1)$, and if it is rational, the distribution is periodic. The idea is to exploit the equidistribution theorem for the sequence of the intersections between integer squares and a straight segment. Nevertheless, some adaptation is needed since the start point is a family, indexed by the grid step $h$, of finite sequences of intersections.

In this section, $d_{1}$ denotes the $L_{1}$-distance.

\subsection{Diagonal sequences of a straight line}

All along the section, we consider a non-vertical straight line $D$ with positive slope (the case where $D$ has a negative slope is similar and leads to the same results). 
For any $k \in \mathbb{Z}$, we define $v_{k}$ the intersection of the line $D$ with the straight line $\Delta_{k}: x+y=k$ (see Figure 6). With a negative slope, we would consider the lines $\Delta_{k}: x-y=k$. Thanks to the modulo-1 equivalence relation, we project

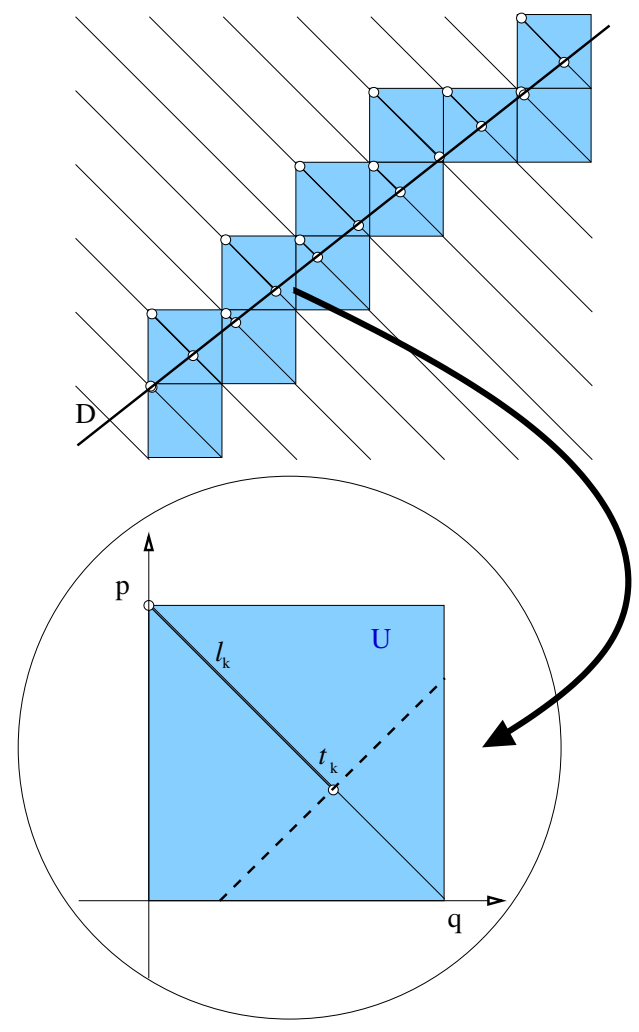

Figure 6: Example of a diagonal sequence for a straight line $D$.

the straight lines $D, \Delta_{k}$ and the points $v_{k}$ on the unit squar ${ }^{3} M^{\prime}=[0,1) \times(0,1]$ ( $M^{\prime}=M=[0,1)^{2}$ if the slope of $D$ is negative). Let us consider the points $p=$ $(0,1)$ and $q=(1,0)$ (when the slope is negative, $p=(0,0)$ and $q=(1,1)$ ). Each line $\Delta_{k}$ projects on the semi-open interval $[p, q)$ while each point $v_{k}$ projects on a point $t_{k} \in[p, q)$. Then, we define the real $l_{k} \in[0,1)$ such that $t_{k}=p+l_{k}(q-p)$. In the sequel, we refer to the sequences $\left\langle v_{k}\right\rangle_{k \in \mathbb{Z}},\left\langle t_{k}\right\rangle_{k \in \mathbb{Z}},\left\langle l_{k}\right\rangle_{k \in \mathbb{Z}}$ as, respectively, the diagonal sequence of $D$, the $2 D$-torus diagonal sequence of $D$ and the $1 D$ torus diagonal sequence of $D$. We also define the corresponding finite sequences with any segment of $D$. Eventually, we say that $[p, q)$ is the torus diagonal related to $D$.

The two following lemmas are obvious but useful for our enumeration of the intersections.

\footnotetext{
${ }^{3}$ We project on $M^{\prime}$ rather than $M$ to ensure that the lines $\Delta_{k}$ are projected on a segment.
} 
Lemma 5.1. Let $D$ be a non-vertical straight line. Then, the $d_{1}$ distance between two consecutive elements of the diagonal sequence of $D$ is equal to 1.

Proof. Putting $v_{k}\left(x_{k}, y_{k}\right)$ and $v_{k+1}\left(x_{k+1}, y_{k+1}\right)$, we derive from the hypotheses $v_{k} \in \Delta_{k}, v_{k+1} \in \Delta_{k+1}$ that $\left(x_{k+1}-x_{k}\right)+\left(y_{k+1}-y_{k}\right)=1$. Besides, for the slope of $D$ is positive, $x_{k+1}-x_{k}$ and $y_{k+1}-y_{k}$ share the same sign. Then, they are both positive and the result holds.

Lemma 5.2. Let $[a, b]$ be a straight segment with irrational slope. For a grid of step $h$, we note $n_{a, b, h}$ the number of grid squares intersected by the segment $[a, b]$. Then

$$
n_{a, b, h} \underset{h \rightarrow 0}{\sim} \frac{d_{1}(a, b)}{h} .
$$

Proof. Since the slope of the segment $[a, b]$ is irrational, it contains at most one grid point. Then, it is plain that the number of grid squares crossed by the segment $[a, b]$ differs from the size of the diagonal sequence of $[a, b]$ by at most 2. Using Lemma 5.1 on the $h$-grid, we conclude straightforwardly.

Proposition 5.1 (Diagonal sequence equidistribution). Let $D$ be a non-vertical straight line whose slope is irrational. Then, the $1 D$-torus diagonal sequence of $D$ is equidistributed over $[0,1)$.

Proof. Let us find the relation that links $l_{k}$ to $l_{k+1}$. From Lemma 5.1, we derive that either $t_{k+1}-t_{k}+p$ (Fig. 7. left), $t_{k+1}-t_{k}+q$ (Fig. 7, center), is collinear to the line $D$. Note that in the case depicted in Fig. 7 right, both vectors are collinear to $D$.
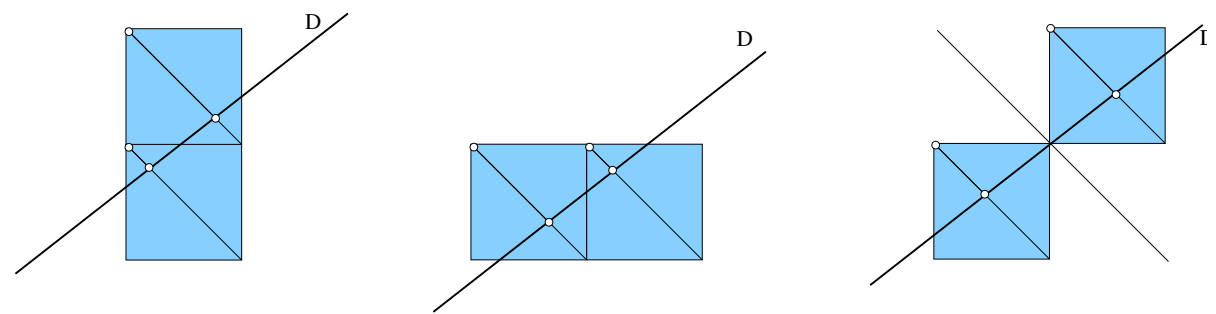

Figure 7: Illustration of the three cases encountered for two consecutive pixels intersected by a straight line.

Let $u$ be the direction vector of $D$ and $s$ the slope of $D$. Setting $\psi=\operatorname{det}(\cdot, u)$, $r=\frac{\psi(p)}{\psi(p)-\psi(q)}=\frac{1}{1+s}$ and noting that $t_{k+1}-t_{k}=\left(l_{k+1}-l_{k}\right)(q-p)$, an elementary calculus yields $l_{k+1}-l_{k}=r$ or $l_{k+1}-l_{k}=r-1$. We derive that the sequence $\left(l_{k}\right)_{k \in \mathbb{Z}}$ is an arithmetic progression on $\mathbb{R} / \mathbb{Z}$ with an irrational common difference $\frac{1}{1+s}$. By the equidistribution theorem, we get that the real $l_{k}$ are equidistributed over $[0,1)$. 


\subsection{Intersection sequence of two straight lines}

Given two non-parallel straight lines projected on the torus, we aim at estimating the number of intersection points. Firstly, we define the intersection sequence.

Let $D_{1}, D_{2}$ be two non-parallel straight lines of $\mathbb{R}^{2}$. Let $\left[p_{1}, q_{1}\right)$ and $\left[p_{2}, q_{2}\right)$ be the torus diagonals related to $D_{1}$ and $D_{2}$. For any $x, y$ in $[0,1)$, the straight lines parallel to $D_{1}$ and $D_{2}$ passing respectively through $p_{1}+x\left(q_{1}-p_{1}\right)$ and $p_{2}+y\left(q_{2}-p_{2}\right)$ intersect in a point $\varphi(x, y)$.

Let $\left\langle l_{i}^{1}\right\rangle,\left\langle l_{j}^{2}\right\rangle$ be the $1 D$-torus diagonal sequences of $D_{1}$ and $D_{2}$. The sequence $\left\langle\varphi\left(l_{i}^{1}, l_{j}^{2}\right)\right\rangle_{i, j \in \mathbb{Z}}$ is called the intersection sequence of $D_{1}$ and $D_{2}$. An Illustration is given in Figure 8, where the two cases with the intersection in and out of the square are represented.
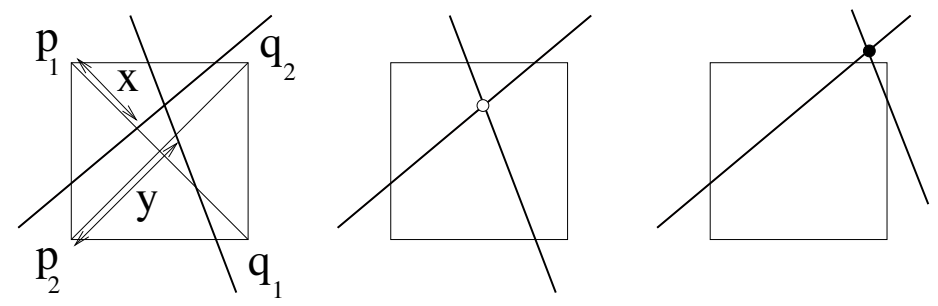

Figure 8: Crossing of straight lines. Left: notations ; Center: intersection (white dot) in the square ; Right: intersection (black dot) out of the square.

Proposition 5.2 (Intersection of two straight lines). Let $D_{1}$ and $D_{2}$ be two non-parallel straight lines of $\mathbb{R}^{2}$ with irrational slopes. Then $\varphi\left([0,1)^{2}\right)$ is a parallelogram so-called intersection set and the proportion of elements in the intersection sequence $\left\langle\varphi\left(l_{i}^{1}, l_{j}^{2}\right)\right\rangle_{(i, j)=(0,0)}^{(i, j)=(m, n)}$ which lie in the square $M=[0,1)^{2}$ tends toward the inverse of the area of the intersection parallelogram as the integers $m$ and $n$ tend toward infinity.

Proof. $D_{1} \oplus[0,1)$ is a strip whose borders are parallel. So is $D_{2} \oplus[0,1)$. By hypothesis, the straight lines $D_{1}$ and $D_{2}$ are not parallel. So, $\varphi\left([0,1)^{2}\right)$ is a parallelogram.

The coordinates $(a, b)$ of the intersection $\varphi(x, y)$ in $\mathbb{R}^{2}$ are obtained by solving a linear system given by the equations of two straight lines parallel to $D_{1}$ and $D_{2}$ passing through points that depend affinely on $x$ and $y$. Then the function $\varphi$ is an affine function in $(x, y)$. As a consequence, the set $\varphi^{-1}(M)=\{(x, y) \in$ $\left.[0,1)^{2} \mid \varphi_{x, y} \in M\right\}$ is measurable. Let define the function

$$
f:(x, y) \in[0,1)^{2} \mapsto \mathbf{1}_{M}(\varphi(x, y)) .
$$

Clearly, $f$ is Riemann integrable. Then the criterion of Weyl-Riemann applies to the pairs $\left(l_{i}^{1}, l_{j}^{2}\right)$ which are equidistributed in $[0,1)^{2}$ (Prop. 5.1):

$$
\sum_{i=0}^{m} \sum_{j=0}^{n} f\left(l_{i}^{1}, l_{j}^{2}\right) \underset{m, n \rightarrow \infty}{\longrightarrow} \int_{0}^{1} \int_{0}^{1} f(x, y) d x d y
$$


This integral of the indicator function is the area of $\varphi^{-1}(M)$ which is a subset of the unit square $M$. Let us show that this area is equal to the inverse of the area of the parallelogram $\varphi\left([0,1)^{2}\right)$. The fact that $\varphi$ is affine induces that it conserves the ratios:

$$
\frac{\operatorname{area}\left(\varphi^{-1}(M)\right)}{\operatorname{area}\left(\varphi^{-1}\left(\varphi\left([0,1)^{2}\right)\right)\right)}=\frac{\operatorname{area}(M)}{\operatorname{area}\left(\varphi\left([0,1)^{2}\right)\right)} .
$$

So, we obtain

$$
\int_{0}^{1} \int_{0}^{1} f(x, y) d x d y=\frac{1}{\operatorname{area}\left(\varphi\left([0,1)^{2}\right)\right.} \text {. }
$$

Let us now compute the area of the codomain of $\varphi$.

Proposition 5.3 (Area of $\varphi\left([0,1)^{2}\right)$ ). Let $D_{1}, D_{2}$ be two straight lines in $\mathbb{R}^{2}$ with distinct irrational slopes $s_{1} \neq s_{2}$. The surface of the intersection set is given by the following formula:

$$
\operatorname{area}\left(\varphi\left([0,1)^{2}\right)\right)=\frac{\left(1+\left|s_{1}\right|\right)\left(1+\left|s_{2}\right|\right)}{\left|s_{2}-s_{1}\right|} .
$$

Proof. The formula is obtained by an analytic calculus of the area of a parallelogram. The vertices of the parallelogram need to be computed to obtain the parallelogram area: let note $c_{i, j}=\varphi(i, j)$ for $i, j \subset\{0,1\}$.

First case: $s_{1} s_{2} \geq 0$. We begin with the subcase $s_{1} \geq 0, s_{2} \geq 0$ (see Fig 9-left).

Firstly we compute the coordinates of the point $c_{0,1}=\varphi(0,1)$, intersection of the straight line parallel to $D_{1}$ including the vertex $p_{1}$ and the straight line parallel to $D_{2}$ including the vertex $q_{1}$ :

$$
x_{c_{0,1}}=\frac{s_{1}-1}{s_{2}-s_{1}} ; \quad y_{c_{0,1}}=\frac{-s_{1}\left(1+s_{2}\right)}{s_{2}-s_{1}} .
$$

Then, the absolute value of the determinant $\operatorname{det}\left(\mathbf{c}_{\mathbf{0}, \mathbf{1}} \mathbf{c}_{\mathbf{0}, \mathbf{0}}, \mathbf{c}_{\mathbf{0}, \mathbf{1}} \mathbf{c}_{\mathbf{1}, \mathbf{1}}\right)$ gives the result.

$$
\operatorname{area}\left(\varphi\left([0,1)^{2}\right)\right)=\frac{\left(1+s_{1}\right)\left(1+s_{2}\right)}{\left|s_{2}-s_{1}\right|} .
$$

When the slopes $s_{1}$ and $s_{2}$ are non-positive, we make a horizontal symmetry to use the previous result. So, the general formula in the case where the two slopes share the same sign is

$$
\operatorname{area}\left(\varphi\left([0,1)^{2}\right)\right)=\frac{\left(1+\left|s_{1}\right|\right)\left(1+\left|s_{2}\right|\right)}{\left|s_{2}-s_{1}\right|} .
$$

Second case: $s_{1} s_{2} \leq 0$. We begin with the subcase $s_{1} \geq 0$ and $s_{2} \leq 0$ (see Fig 9 right). 

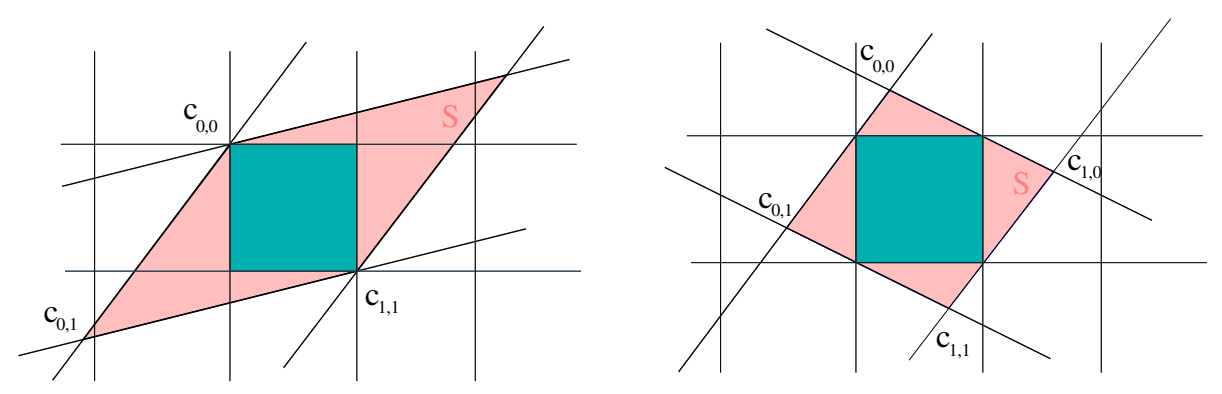

Figure 9: Scheme of parallelogram $S$ used for the edge intersection asymptotic number computation. Left: slopes in the same quadrant; right: slopes in distinct quadrant.

Then,

$$
\begin{aligned}
& x_{c_{0,0}}=\frac{-1}{s_{1}-s_{2}} ; \quad y_{c_{0,0}}=\frac{-s_{2}}{s_{1}-s_{2}} ; \\
& x_{c_{0,1}}=\frac{-s_{2}}{s_{1}-s_{2}} ; \quad y_{c_{0,1}}=1-\frac{s_{2} s_{1}}{s_{1}-s_{2}} ; \\
& x_{c_{1,0}}=\frac{-s_{1}}{s_{1}-s_{2}} ; \quad y_{c_{1,0}}=\frac{s_{1} s_{2}}{s_{1}-s_{2}} .
\end{aligned}
$$

Then, the desired result is given by the absolute value of the determinant $\operatorname{det}\left(\mathbf{c}_{\mathbf{0}, \mathbf{0}} \mathbf{c}_{\mathbf{0}, \mathbf{1}}, \mathbf{c}_{\mathbf{0}, \mathbf{0}} \mathbf{c}_{\mathbf{1}, \mathbf{0}}\right)$.

$$
\begin{aligned}
\operatorname{area}\left(\varphi\left([0,1)^{2}\right)\right) & =\left|\frac{\left(1+s_{1}\right)\left(1-s_{2}\right)}{s_{1}-s_{2}}\right| \\
& =\frac{\left(1+\left|s_{1}\right|\right)\left(1+\left|s_{2}\right|\right)}{\left|s_{2}-s_{1}\right|} .
\end{aligned}
$$

The second subcase, $s_{1} \leq 0$ and $s_{2} \geq 0$ is obtained by inverting $D_{1}$ and $D_{2}$ which does not change the formula.

We now extend the previous result to the edges of a polygon when the grid step tend toward zero.

Proposition 5.4 (Two polygon edge formula). Let E, $F$ be two edges of a polygon $P$ in $\mathbb{R}^{2}$ with distinct irrational slopes. For $h>0$, we note $i(E, F, h)$ the number of intersections of the edge projections on the torus $(\mathbb{R} / h \mathbb{Z})^{2}$. Then,

$$
i(E, F, h) \underset{h \rightarrow 0}{\sim} \frac{n_{E, h} n_{F, h}}{\operatorname{area}\left(S_{E, F}\right)}
$$

where $S_{E, F}$ is the intersection set and $n_{E, h}$, respectively $n_{F, h}$, is the number of boundary grid squares crossed by the edge $E$, respectively $F$. 
Proof. Let $D_{E}$ and $D_{F}$ be the straight lines parallel to $E$ and $F$ and passing through the origin. As the slope of the edges $E$ and $F$ are irrational, we derive from Prop. 5.2 that the proportion of elements in the intersection sequence of $D_{E}$ and $D_{F}$ that lie in the unit square tends toward 1/area $\left(S_{E, F}\right)$. Besides, there are $n_{E, h}$ boundary grid squares crossed by the edge $E$ and $n_{F, h}$ other boundary grid squares crossed by the edge $F$. Then, there are $n_{E, h} n_{F, h}$ pairs of boundary grid squares that can yield an intersection. We derive that the number of elements in the intersection sequence of $D_{E}$ and $D_{F}$ that lie in the unit square is equivalent to $n_{E, h} n_{F, h} / \operatorname{area}\left(S_{E, F}\right)$.

The second part of the proof is here to show the equivalence between the straight line case and the case of segments when the grid step tends toward 0 .

We assume that $E$ and $F$ are parameterized by $E=\{a+t b \mid t \in[0,1]\}$ and $F=\{c+u d \mid u \in[0,1]\}$ where $a, b, c, d \in \mathbb{R}^{2}$. Then, finding the intersections of the edge projections on $(\mathbb{R} / h \mathbb{Z})^{2}$ amounts to solve $\{a / h+t b\}=\{c / h+u d\}$ where $\{\cdot\}$ denotes the fractional part and $t, u$ lie in $[0,1 / h]$. It is equivalent to solve $k+e+t b=u d$ where $k \in \mathbb{Z}^{2}, e \in[0,1)^{2}$ and $t, u$ lie in $[0,1 / h]$. Projecting $e$ on the basis $(b, d)$, gives $k+t b=u d$ where $t \in\left[0,1 / h+e_{b}\right), u \in\left[0,1 / h+e_{d}\right)$ and $e_{b}$ and $e_{d}$ are bounded. The latter constraint equation also expresses as $\{t b\}=\{u d\}$ where $t \in\left[0,1 / h+e_{b}\right), u \in\left[0,1 / h+e_{d}\right)$. Finally, we fall back to the problem of finding the intersection sequence elements of $D_{E}$ and $D_{F}$ that lie in the unit square. Then, we get the result from the first part of the proof.

Remark 5.1. The case where at least one of the slopes is rational leads to replace area $\left(S_{E, F}\right)$ by a discrete approximation depending on the rational denominators in Formula 4

From the formula (3) and Prop. 5.4 it can be said that two almost parallel edges lead to a large area parallelogram and few intersections. Conversely, if one edge is almost horizontal and the other is almost vertical, the number of intersections is close to the supremum $n_{E, h} n_{F, h}$.

\subsection{Modulo-1 polygon intersection set}

Theorem 1 (Number of intersections for a polygon). Let $P$ be a polygon whose edges $\left(e_{i}\right)_{i=1}^{m}$ have distinct irrational slopes. Then, when $h$ tends toward 0 , the number of intersections is equivalent to

$$
\frac{1}{h^{2}} \sum_{i<j \leq m} \frac{\left\|e_{i}\right\|_{1}\left\|e_{j}\right\|_{1}\left|s_{e_{j}}-s_{e_{i}}\right|}{\left(1+\left|s_{e_{i}}\right|\right)\left(1+\left|s_{e_{j}}\right|\right)}
$$

Proof. We apply Prop. 5.4 to each pair of edges.

The result on the intersections can be used is the Gauss digitization upper bound exhibited in Section 3.2 . 
Corollary 5.1 (Polygon digitization combinatorics). Let $P$ be a polygon whose edges $\left(e_{i}\right)_{i=1}^{m}$ have distinct irrational slopes and let $n_{h}$ be cardinal of the toggling boundary in the h-grid. The number of Gauss digitizations of the polygon $P$ in a grid of step $h$ is a $O\left(n_{h}^{2}\right)$ when $h$ tends toward 0.

Proof. Formula (5) has to be transformed to make the toggling boundary cardinal appear. As the intersection sets all contain a unit square, their areas are greater than or equal to 1 , that is, for any pair $i \neq j$,

$$
\frac{\left(1+\left|s_{e_{i}}\right|\right)\left(1+\left|s_{e_{j}}\right|\right)}{\left|s_{e_{j}}-s_{e_{i}}\right|} \geq 1 .
$$

Then,

$$
\begin{aligned}
\frac{1}{h^{2}} \sum_{i<j \leq m} \frac{\left\|e_{i}\right\|_{1}\left\|e_{j}\right\|_{1}\left|s_{e_{j}}-s_{e_{i}}\right|}{\left(1+\left|s_{e_{i}}\right|\right)\left(1+\left|s_{e_{j}}\right|\right)} \\
\quad \leq \frac{1}{h^{2}} \sum_{i<j \leq m}\left\|e_{i}\right\|_{1}\left\|e_{j}\right\|_{1} \\
\leq \frac{1}{2}\left(\frac{\sum_{i}\left\|e_{i}\right\|_{1}}{h}\right)^{2} .
\end{aligned}
$$

The term $\sum_{i}\left\|e_{i}\right\|_{1} / h$ is asymptotically equivalent to the toggling boundary cardinal $n_{h}$. Therefore, the intersection number is a $O\left(n_{h}^{2}\right)$.

Using this polygon intersection asymptotic upper bound $O\left(n_{h}^{2}\right)$ in Formula (1) leads to the result.

\section{Conclusion}

We present in this paper two general upper bounds on the number of digital objects obtained from continuous object up to a translation. The first one is exponential in the cardinal of the the so-called toggling boundary and a generic example reaching this bound is given. The second one is based on the correspondence between the dual connected-components and number of intersections of the tore-projected continuous boundary.

The intersection based upper bound is then derived in two cases. In the convex case, it yields to an upper bound quadratic in the perimeter pixel size. An example of a convex object is given where the set of translations corresponding to a given digitization is not connected. Eventually in the polygon case, provided the slopes are irrational, an upper bound depending on the lengths and slopes of the edges is obtained. This upper bound is asymptotically quadratic in the toggling boundary cardinal.

The perspectives of this work are the following: Firstly to extend the polygonal case method to splines since the equidistribution theorem also applies in this case; then to propose an efficient algorithm for the digital object generation, to 
study the combinatorics of the digital object set under the rigid transformations (including rotations) and to extend the results to 3D.

\section{Appendix}

\section{A Convex sets}

The proof of Lemma A.3 relies on the two following lemmas about convex sets that seem obvious at the first glance. Nevertheless, since we did not find any result related to these lemmas in the literature, we provide our own justifications of the two statements.

Lemma A.1 (Chords of convex sets). Let $[a, b]$ be a chord of the boundary $\Gamma$ of a closed convex set $S$. If $[a, b] \nsubseteq \subseteq$, then $(a b) \cap S=[a, b]$ and $(a b) \cap \Gamma=\{a, b\}$.

Proof. Since $[a, b] \nsubseteq \mp$, the line $(a b)$ does not support $S$ at any point (the notion of supporting line of a convex set is exposed for instance in [2]). So, there exists two supporting lines of $S$ at $a$ and $b$ that cross the line $(a b)$. Then, $(a b) \cap S$ is included in $[a, b]$. Let $c \in[a, b] \cap \Gamma, c \neq a$. Applying the first part of the proof to the chord $[a, c]$, we derive that $(a c) \cap S \subseteq[a, c]$ and, since $b \in(a c) \cap S$, we conclude that $b=c$.

Lemma A.2 (Cuts of convex sets). Let $a, b$ be two points of the boundary $\Gamma$ of a closed convex set $S$. If $[a, b] \not \subset \Gamma$, then the open curve segments of $\Gamma, \sigma_{1}, \sigma_{2}$, whose extremities are $a$ and $b$ are included in distinct open half-planes bounded by the line $(a b)$.

Proof. Let $H^{-}$and $H^{+}$be the two open half-planes bounded by $(a b)$. Since $[a, b] \nsubseteq \Gamma$, from Lemma A.1 $(a b) \cap \Gamma=\{a, b\}$. Thereby, by connectivity, either $\sigma_{1} \subset H^{-}$or $\sigma_{1} \subset H^{+}$and $\sigma_{2} \subset H^{-}$or $\sigma_{2} \subset H^{+}$. Suppose for instance that $\sigma_{1} \subset H^{-}$and $\sigma_{2} \subset H^{-}$. Then, $S$, which is the connected subset of $\mathbb{R}^{2}$ bounded by $\sigma_{1} \cup \sigma_{2} \cup\{a, b\}$ is included in $H^{-} \cup(a b)$ and, since $(a b) \cap S=[a, b]$ from Lemma A.1 $[a, b] \subset \Gamma$ : contradiction.

Lemma A.3 (Intersection of two segments of a convex curve). Let $\Gamma$ be a Jordan curve whose interior is convex. Let $\Gamma_{1}$ and $\Gamma_{2}$ two disjoint closed segments of the curve $\Gamma$ and $\tau$ a vector of $\mathbb{R}^{2}$. Then the intersection of $\Gamma_{1}$ and $\tau+\Gamma_{2}$ is composed of none, one, two points or a line segment.

Proof. Let $p, q$ be two distinct points in $\Gamma_{1} \cap\left(\tau+\Gamma_{2}\right)$ if such a pair exists. We denote by $\Sigma_{1}$ the open segment of $\Gamma_{1}$ between $p$ and $q$. Alike, $\Sigma_{2}$ is the open segment of $\Gamma_{2}$ between $-\tau+p$ and $-\tau+q$. We set $\bar{\Sigma}_{1}=\Sigma_{1} \cup\{p, q\}$ and $\bar{\Sigma}_{2}=\Sigma_{2} \cup\{p, q\}$. Firstly, we prove that $\Sigma_{1} \cup\left(\tau+\Sigma_{2}\right)$ is a straight line segment whenever it contains more than two points. First case: $\Sigma_{1} \cup\left(\tau+\Sigma_{2}\right) \subseteq(p q)$. Then, since $\bar{\Sigma}_{1}$ and $\bar{\Sigma}_{2}$ are connected and $\Gamma$ is simple, $\bar{\Sigma}_{1}=\bar{\Sigma}_{2}=[p, q]$. Second case: $\exists x \in\left(\Sigma_{1} \cup\left(\tau+\Sigma_{2}\right)\right) \backslash(p q)$. For instance, we assume $x \in \Sigma_{1} \backslash(p q)$. By Lemma A.2, $\Sigma_{1}=\Gamma \cap H_{1}$, where $H_{1}$ is the open half-plane bounded by the 
line $(p q)$ and containing $x$, and $-\tau+p$ is in $\mathbb{R}^{2} \backslash H_{1}$. Then, it can easily be seen that $-\tau+H_{1}$ is the open half-plane bounded by the line joining $-\tau+p$ and $-\tau+q$ and including $p$. Thanks to Lemma A.2 we derive that $\Sigma_{2}$ does not intersect $-\tau+H_{1}$. Thus, $\tau+\Sigma_{2}$ does not intersect $H_{1}$. In particular, $\left(\tau+\Sigma_{2}\right) \cap \Sigma_{1}=\emptyset$. This achieves the first part of the proof. Now, let $r$ be a point in $\Gamma_{1} \cap\left(\tau+\Gamma_{2}\right)$ which is not in $\Sigma_{1}$ (if such a point exists). For instance, $p$ belongs to the segment of $\Gamma_{1}$ between $q$ and $r$. Then, the first part of the proof, applied to the points $q$ and $r$, implies that $\Gamma_{1} \cap\left(\tau+\Gamma_{2}\right)$ includes the segments $[q, r]$.We straightforwardly concludes that either the intersection of $\Gamma_{1}$ and $\tau+\Gamma_{2}$ is composed of at most two points or it is a line segment.

\section{B Examples and counterexamples}

\section{B.1 Building examples without proper congruent digiti- zations in the image of the dual}

Let $u$ and $v$ be two vectors in $[0,1)^{2}$ such that the sets $u+S$ and $v+S$ have distinct but congruent digitizations. Then, there exists an integer vector $w$, $w \neq 0$, such that $(u+S) \cap \mathbb{Z}^{2}=w+\left((v+S) \cap \mathbb{Z}^{2}\right)=(w+v+S) \cap \mathbb{Z}^{2}$. Let $p$ be a point in the digitization core. Then, $p \in(u+S) \cap \mathbb{Z}^{2}$ and $p \in(v+S) \cap \mathbb{Z}^{2}$. Therefore, $w+p \in(u+S) \cap \mathbb{Z}^{2}$ and $-w+p \in(v+S) \cap \mathbb{Z}^{2}$, which can be rewritten as $p \in((-w+u)+S) \cap \mathbb{Z}^{2}$ and $p \in((w+v)+S) \cap \mathbb{Z}^{2}$. Then, at least one of the vectors $w+v$ or $-w+u$ has one of its coordinates which is negative. We derive that if there exists a point in the digitization core which is maximal in $S$ for both coordinates then there is no proper congruent digitizations in the dual. An example with such a maximal point in the digitization core is provided in Fig. 10 .

\section{B.2 Building toric partitions in one-to-one correspondence with the power set of the toggling boundary}

In this section, we exhibit a way to modify the boundary of the set $S$ in order to ensure that any subset of the toggling boundary is represented in the dual. To do so, we move along $\mathcal{B}$, ordered in the same way as in Def. 1 . Then, a new boundary is built thanks to the approximations of the Hilbert filling curve: the segment of $\Gamma$ intersecting the $n$-th cell of $\mathcal{B}$ is replaced by a $n$-th approximation of the Hilbert filling curve $H_{n}$ (extended at its extremities to ensure the continuity of the boundary): see Fig. 10. We consider the family of binary partitions $\mathcal{P}_{n}$ of the unit square that comes with the curves $H_{n}$. We claim that each curve $H_{n+1}$ crosses each cell of the partition $\bigwedge_{i=1}^{n} \mathcal{P}_{i}$ so that the size of the final torus partition is $2^{N}$ where $N$ is the cardinal of the toggling boundary. To justify our claim, we divide the unit square in a family of $2^{n} \times 2^{n}$ small squares $\left(K_{i, j}^{n}\right)_{1 \leq i, j \leq 2^{n}}(n \geq 0)$ whose sizes are $\frac{1}{2^{n}} \times \frac{1}{2^{n}}$. It can be seen that on the one hand, the Hilbert curve $H_{n}$ passes through the center of each of the squares $K_{i, j}^{n}$ and, on the other hand, does not intersect any of the interior of the 


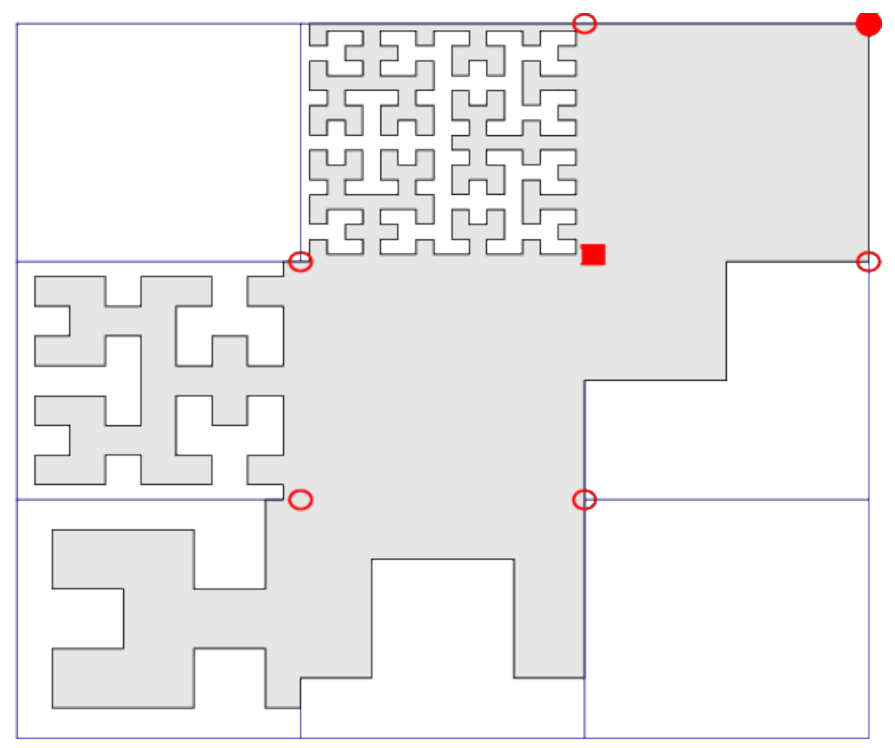

Figure 10: (Color online) The boundary of the depicted set is obtained by connecting $n$-th approximations of the Hilbert filling curve, $0 \leq n \leq 4$. Red square and red disc: digitization core. Red circle: the five points in the toggling boundary. The red disc is both in the grid boundary and in the digitization core. Furthermore, it is maximal for both coordinates which ensures that there is no proper congruent digitizations in the image of the dual. The set $S$ has $2^{5}$ digitizations.

squares $K_{i, j}^{n+1}$ ( $H_{0}$ is just the center of the unit square). Thereby, the partition $\bigwedge_{i=1}^{n} \mathcal{P}_{i}$ is coarser than the partition $\left\{K_{i, j}^{n+1} \mid 1 \leq i, j<n\right\}$ (the boundaries of the squares $K_{i, j}^{k}$ are assigned to the cells so as to coincide with $\mathcal{P}_{n}$ ). Since $H_{n+1}$ passes through the center of each of the squares $K_{i, j}^{n+1}$, it passes in each cell of $\bigwedge_{i=1}^{n} \mathcal{P}_{i}$ which gives the claim.

\section{References}

[1] Baudrier, É., Mazo, L.: Curve digitization variability. In: Normand, N, Guédon, J, Autrusseau, F (eds.) Discrete Geometry for Computer Imagery. DGCI 2016, Lecture Notes in Computer Science, vol. 9647, pp. 59-70. Springer, Heidelberg, IAPR (2016) doi: 10.1007/978-3-319-32360-2_5

[2] Berger, M.: Geometry (vols. 1) (1987)

[3] Dorst, L., Smeulders, A.W.M.: Discrete Representation of Straight Lines. IEEE Trans. Pattern Anal. Mach. Intell. 6(4), 450-463 (jul 1984), http://ieeexplore.ieee.org/lpdocs/epic03/wrapper.htm? arnumber $=4767550$ 
[4] Feschet, F., Tougne, L.: An approach for the estimation of the precision of a real object from its digitization. Discrete Applied Mathematics 139(1-3): 51-63 elsevier, 2004. doi: 10.1016/j.dam.2002.11.009

[5] Heath-Brown, D.: Lattice points in the sphere. Number theory in progress 2, 883-892 (1997)

[6] Huxley, M.N.: The number of configurations in lattice point counting I. Forum Mathematicum 22(1), 127-152 (2010)

[7] Huxley, M.N., Žunić, J.D.: On the number of digitizations of a disc depending on its position. In: Combinatorial Image Analysis, 10th Int Workshop, IWCIA 2004, New Zealand. pp. 219-231 (2004) doi: 10.1007/978-3-54030503-3_17

[8] Huxley, M.N., Žunić, J.D.: Different digitisations of displaced discs. Found of Comput Math 6(2), 255-268 (2006), doi: 10.1007/s10208-005-0177-y

[9] Huxley, M.N., Žunić, J.D.: The number of $N$-point digital discs. IEEE Trans. Pattern Anal. Mach. Intell. 29(1), 159-161 (2007), doi: 10.1109/TPAMI.2007.250606

[10] Huxley, M.N., Žunić, J.D.: The number of different digital $N$-discs. J Math Imaging and Vision 56(3), 403-408 (2016), doi: 10.1007/s10851-016-0643-y

[11] Mazo, J.E., Odlyzko, A.M.: Lattice points in high-dimensional spheres. Monatshefte für Mathematik 110(1), 47-61 (1990) doi: 10.1007/BF01571276

[12] Mazo, L., Baudrier, É.: Object digitization up to a translation. J Computer and System Science (2017), doi: 10.1016/j.jcss.2017.08.001

[13] Mazo, L., Baudrier, É.: Study on the digitization dual combinatorics and convex case. In: W.G. Kropatsch, N.M. Artner, I. Janush (Eds), Discrete Geometry for Computer Imagery. DGCI 2017, Lecture Notes in Computer Science, vol. 10502, pp. 363-374, Springer, Heidelberg, IAPR (2017), doi: 10.1007/978-3-319-66272-5

[14] Nagy, B.: An algorithm to find the number of the digitizations of discs with a fixed radius. Electronic Notes in Discrete Math 20, 607-622 (2005), doi: 10.1016/j.endm.2005.04.006

[15] Ngo, P., Kenmochi, Y., Passat, N. and Talbot, H.: Combinatorial structure of rigid transformations in 2D digital images Computer Vision and Image Understanding, 117 (4), 393-408 (2013). doi: 10.1016/j.cviu.2012.08.014

[16] Nouvel, B., Rémila, É.: Configurations induced by discrete rotations: periodicity and quasi-periodicity properties. Discrete Applied Mathematics, 147(2), 325-343 (2005) doi: 10.1016/j.dam.2004.09.018 
[17] Pluta, K., Romon, P., Kenmochi, Y. and Passat, N.: J Math Imaging and Vision, 59(1), 84-105 (2017) doi: 10.1007/s10851-017-0706-8

[18] Weyl, H.: Über die Gleichverteilung von Zahlen mod. Eins [On the distribution of numbers modulo one]. Math. Ann. (in German). 77 (3): 313-352, (1916), doi:10.1007/BF01475864

[19] Žunić, J.D.: On the number of digital discs. J Math Imaging and Vision 21(3), 199-204 (2004), doi: 10.1023/B:JMIV.0000043736.15525.ed

[20] Žunić, J.D.: On the number of ways to occupy $n$ lattice points by balls in $d$-dimensional space. J Number Theory 110(2), 396-402 (2005) doi: $10.1016 /$ j.jnt.2004.08.004 